\title{
Supplementation of dietary germanium biotite enhances induction of the immune responses by foot-and-mouth disease vaccine in cattle
}

\author{
Myunghwan Jung', Min-Kyoung Shin', Seung-Bin Cha', Seung Won Shin', Anna Yoo', Won-Jung Lee',
} Hong-Tae Park', Jong-Hyeon Park'2, Byounghan Kim², Yeon-Kwon Jung ${ }^{3}$ and Han Sang Yoo ${ }^{1,4^{*}}$

\begin{abstract}
Background: After the recent outbreak of foot-and-mouth disease (FMD) in Korea, a vaccination policy has been applied to control the disease. In addition, several non-specific immune stimulators have been used without any scientific evidence that they would enhance the immune response after FMD vaccination and/or protect against FMD. Based on the current situation, the aim of this study was to evaluate the effect of the non-specific immune stimulator germanium biotite on FMD vaccination and immune responses in cattle. To achieve our goal, immune responses to FMD vaccination, such as levels of $\lg G$ and $\lg A$, antibody duration, and virus-neutralizing titers were investigated after germanium biotite feeding. The PBMC typing and proliferative response after stimulation with mitogens, the cytokines expression level of PBMC, and the lysozyme activity in the serum were measured to evaluate the immune enhancing effects of germanium biotite following its administration.
\end{abstract}

Results: Following the first vaccination, high level of IgG (at 4 weeks) and IgA (at 2 and 31 weeks) titers in serum and saliva were observed in the germanium biotite-feeding group $(p<0.05)$. The germanium biotite group also showed high and longstanding inhibition percentage value in ELISA assay at 31 weeks $(p<0.05)$. Generally, higher virus-neutralizing antibody titers were observed in the feeding group at 20 and 31 weeks after vaccination. Following the feeding germanium biotite, the germanium biotite group showed increased subpopulation of $\mathrm{CD}^{+}$lymphocytes and $\mathrm{MHC} \mathrm{I}^{+} \mathrm{II}^{+}$cells in PBMCs at 23 week, responding to stimulation of ConA. The levels of IFN- $\gamma$ (at 3 and 8 weeks), IL-1a (at 3, 11, and 23 weeks), IL-1 (at 3, 8, and 11 weeks), and IL-4 (at 8 and 11 weeks) gene expression were also significantly increased in the feeding group $(p<0.01$ and $p<0.05)$. Feeding with germanium biotite increased the lymphocytes' proliferative response to the stimulation of ConA and LPS at 23 weeks and lysozyme activity at 9 weeks after feeding.

Conclusions: These results suggest that germanium biotite feeding could increase the protection against FMD virus infection via the induction of higher humoral and cellular immune responses in cattle.

Keywords: FMD, Germanium biotite, Immune responses, Cattle

\footnotetext{
*Correspondence: yoohs@snu.ac.kr

1 Department of Infectious Diseases, College of Veterinary Medicine, Seoul

National University, Seoul 151-742, South Korea

${ }^{4}$ Institute of Green Bio Science and Technology, Seoul National University,

Pyeongchang 232-916, South Korea

Full list of author information is available at the end of the article
} 


\section{Background}

Foot-and-mouth disease (FMD) is a highly contagious and economically important disease that affects clovenhoofed animals, and it is characterized by appetite loss, an increase in body temperature, and vesicles in the mouth, tongue, hooves, and nipples [1,2]. The disease is caused by the FMD virus (FMDV), which is a small, icosahedral, non-enveloped RNA virus classified within the Aphthovirus genus as a member of Picornaviridae. The clinical severity of the disease varies with the strain of FMDV, infection dose, species, and individual susceptibility of the host [3]. The disease can be transmitted via direct or indirect contact between FMDV-infected animals and susceptible animals [2].

Due to its high mutation rate, this virus exists as seven distinct serotypes (O, A, C, Asia 1, South African territories [SAT]1, SAT2, and SAT3) as well as numerous and constantly evolving subtypes, which shows a spectrum of antigenic diversity $[2,3]$. Therefore, one major problem in controlling FMD is antigenic variation, as infection or vaccination with one FMDV serotype does not protect against other serotypes, and it may even fail to protect fully against other subtypes within the same serotype [4-6]. This problem has been raised by the experimental and field data of previous researchers on vaccination, including both single and multivalent vaccines $[3,7,8]$.

Despite the powerful effects of vaccines, which have mitigated enormous FMD outbreaks, the current vaccines have many problems to overcome such as the long time required to induce antibodies and short duration of immunity when applying them to emergency and routine vaccination programs [6-9]. Therefore, strategies to improve the immune response to vaccination have included using higher vaccine doses or increasing the number of doses, using different routes of administration, accelerating the dosing schedule, and using adjuvants such as antigen delivery systems and various immunostimulators [10].

The immune response against FMDV is related to circulating humoral antibody titers; this is considered the most important factor in conferring protection against FMD [4]. The importance of cell-mediated immunity also has been recognized in the induction of humoral immunity and the clearance of FMDV. T-cell responses mediated by $\mathrm{CD}^{+}$cells are required for protective immunity against FMDV as they participate in the production of antiviral antibodies [11]. Subsets of the $\mathrm{CD}^{+}$major histocompatibility complex (MHC) class II-restricted T-cells respond to activation by antigen-presenting cells and antigens by producing a $\mathrm{T}$ helper type 1 (Th1, IFN- $\gamma$ ) and T helper type 2 (Th2; IL-4, IL-5, and IL-13) responses [12]. Several studies have also demonstrated the presence of FMDspecific MHC II-restricted responses in cattle and pigs [11-13]. The antiviral responses of $\mathrm{CD}^{+} \mathrm{T}$-cells were also detected following FMDV infection in previous studies
$[14,15]$. These antiviral responses were through direct cytotoxicity or release of cytokines such as IFN- $\gamma$ following vaccination, and that the antiviral responses were almost 100 times higher following re-stimulation [15]. MHC class I-restricted CD8 ${ }^{+}$T-cells also showed specific immune response to FMDV as memory cells; however, the correlation between FMDV-specific $\mathrm{CD} 8^{+} \mathrm{T}$-cell recognition and protection remains to be defined $[14,15]$. In addition, the innate immune systems, as well as adaptive immune, play an important role in immune responses to FMDV infection $[16,17]$. The innate immune system, characterized by nonspecific responses, is associated with early protection against FMD and is involved in the formation of adaptive immune response to FMDV infection $[16,17]$.

Germanium biotite, as a well-known feed supplement, is a common phyllosilicate mineral that contains potassium, magnesium, iron, aluminum, and silicate. It has been reported that the effects of non-specific immune stimulating of biotite are associated with the immune cells being stimulated by silicates [18-20]. Fibrogenic silicates $\left(\mathrm{SiO}_{2}\right)$ activated proinflammatory macrophages, and aluminosilicates $\left(\mathrm{Al}_{2} \mathrm{SiO}_{5}\right)$ improved immune-cell differentiation [21]. Aluminosilicates act as a nonspecific immunostimulator that is similar to a superantigen [22]. Superantigens are a class of extremely potent $\mathrm{T}$-cell mitogens, and have a high affinity to regions of MHC II molecules [23]. Indeed, proinflammatory macrophages, which belong to MHC class II antigen-presenting cells, are activated by fibrogenic silicate particulates [21]. Thus, previous studies have suggested that germanium biotite has a potential as a new supplement for immunostimulators, prophylactic agents, and remedial agents [20].

Since the devastating FMD outbreak in Korea at the end of 2010, cattle, pigs, and some small ruminants have been vaccinated with a trivalent FMD vaccine (O1 Manisa, A Malaysia 97, and Asia1 Shamir) at least 6 protective dose $50 \%\left(\mathrm{PD}_{50}\right)$. [24]. To improve the vaccine efficacy, several non-specific immune stimulators have been used in Korea without any scientific understanding of their effects. Therefore, as a first step to understanding the immunological mechanism and improve the vaccine's efficacy, we examined the effects of a non-specific immune stimulator, germanium biotite, in relation to the FMD vaccine in cattle.

\section{Results}

\section{Antibody responses to FDMV}

In the analysis of the duration of antibody levels and the secretion of IgA after FMD vaccination, the inhibition percentage (PI) values started to increase with the first vaccination, and the values were significantly increased by a booster vaccination at 4 weeks, with the highest PI values at 10 weeks, and this continued steadily up to 28 weeks, regardless of whether the germanium biotite was administered. However, the values decreased at 31 weeks in the 
control group when the re-booster vaccination was required, while the values continued at 31 weeks in the germanium biotite group (Figure 1A).

FMDV-specific IgA in the saliva of the germanium biotite group was generally higher than in the control group, although there were no significant differences between the groups at some points during this experiment (Figure 1B). However, IgA could not be detected in the feces of the two groups with the kit used (data not shown).

In the analysis of the early response of IgG, both groups showed a steady increase in the IgG antibody level up to 3 weeks after vaccination; after this point, the level decreased (Figure 1C). The IgG level in the germanium biotite group was higher than in the control group, although the difference was not significant. However, there was a significantly higher IgG antibody level at 4 weeks after vaccination when the booster vaccination was required $(p<0.05$, Figure $1 C)$.

\section{Virus neutralization antibody against the heterologous FMDV strain}

As shown in Table 1, the sera of the control group had a titer of less than 16 at both points. On the other hand, while the germanium biotite group had a VN titer of 16 or less at 20 weeks after vaccination, the $\mathrm{VN}$ titer in the group was found to have increased at 31 weeks after vaccination.

\section{Analysis of peripheral blood mononuclear cell subpopulation}

Changes in the subpopulations of five different lymphocytes were analyzed based on the administration of the germanium biotite. At 23 weeks, $\mathrm{CD} 4^{+}$lymphocytes and $\mathrm{MHCI}^{+} \mathrm{II}^{+}$cells in the germanium biotite group were significantly higher than in the control group $(p<0.01)$, while $\mathrm{CD} 79 \mathrm{a}^{+}, \mathrm{CD}^{+}$, and $\mathrm{CD}^{+}$lymphocytes exhibited no differences between the two groups (Table 2).

\section{Analysis of cytokine gene expression}

The gene expression of IFN- $\gamma$, IL- $1 \alpha$, IL- $1 \beta$, and IL-4 was significantly higher in the germanium biotite group during this experiment (Figure 2). The significant high levels of IFN- $\gamma$ were determined in the germanium biotite group at 3 and 8 weeks, compared to the control $(p<0.01)$. In case of IL- $1 \alpha$ and IL-1 $\beta$, significant differences were observed at 3,11 , and 23 weeks and at 3,8 , and 11 weeks, respectively $(p<0.05$ and $p<0.01)$. The germanium biotite group also showed significant high levels of IL-4 at 8 and 11 weeks, compared to the control $(p<0.01)$. However, there were no noticeable differences in the expression levels of IL- 6 and IL-10 between the two groups although a significant difference in IL-6 expression was observed at 3 week. The germanium biotite feeding induced a significant increase in IFN- $\gamma$ gene expression at a relatively early stage and in IL-4 at a late stage, while increases in IL- $1 \alpha$ and IL- $1 \beta$ gene expression were observed throughout the experimental period.

\section{Lymphocyte proliferation}

Higher lymphocyte proliferation in the germanium biotite group was observed by LPS and ConA stimulation at 23 weeks compared to the control (Figure 3A, $p<$ 0.05).

\section{Lysozyme activity}

Of the three points at which the lysozyme activity was measured in the serum, significantly higher lysozyme activity was observed in the germanium biotite group at 9 weeks after starting the feeding (Figure 3B, $p<0.05$ ). The increase was observed at a relatively early stage after germanium biotite feeding.

\section{Discussion}

The current FMD vaccines that are used in cattle need to be administered every 6 months, in accordance with the duration of the antibody, to protect against the disease $[5,6,25,26]$. The diversity of FMD serotypes has also raised issues related to the efficacy of the current FMD vaccines. Furthermore, there is considerable argument about whether the trivalent vaccines show protective abilities against the Andong strain through the crossreactivity of antibodies induced by trivalent vaccination [24]. Based on the current knowledge of FMD vaccines, the need for additional materials and/or methods has been raised with a view to overcoming the problem. Therefore, non-specific immune stimulators have received attention as a possible solution to this issue. In the disastrous FMD outbreak in Korea in 2010 [24], many non-specific immune stimulators were used without any scientific evidence that they would enhance protection against FMDV infection. Although non-specific immune stimulators have been used, their efficiency is still controversial. Therefore, we evaluated the effect of a non-specific immune stimulator, germanium biotite, for FMD vaccination in cattle.

Previous studies reported that a re-booster vaccination was needed 6 months after the booster vaccination based on the decrease of antibody titer against FMDV $[5,6,25,26]$. Coinciding with these previous studies, this study observed a decreased PI value in the control group at 31 weeks. However, the germanium biotite group showed a continued high level of PI value for 31 weeks. In order to elevate the antibody level, a booster vaccination was needed 4 weeks after the first vaccination. At that time, the germanium biotite group showed a high level of IgG antibody compared to the control. This 

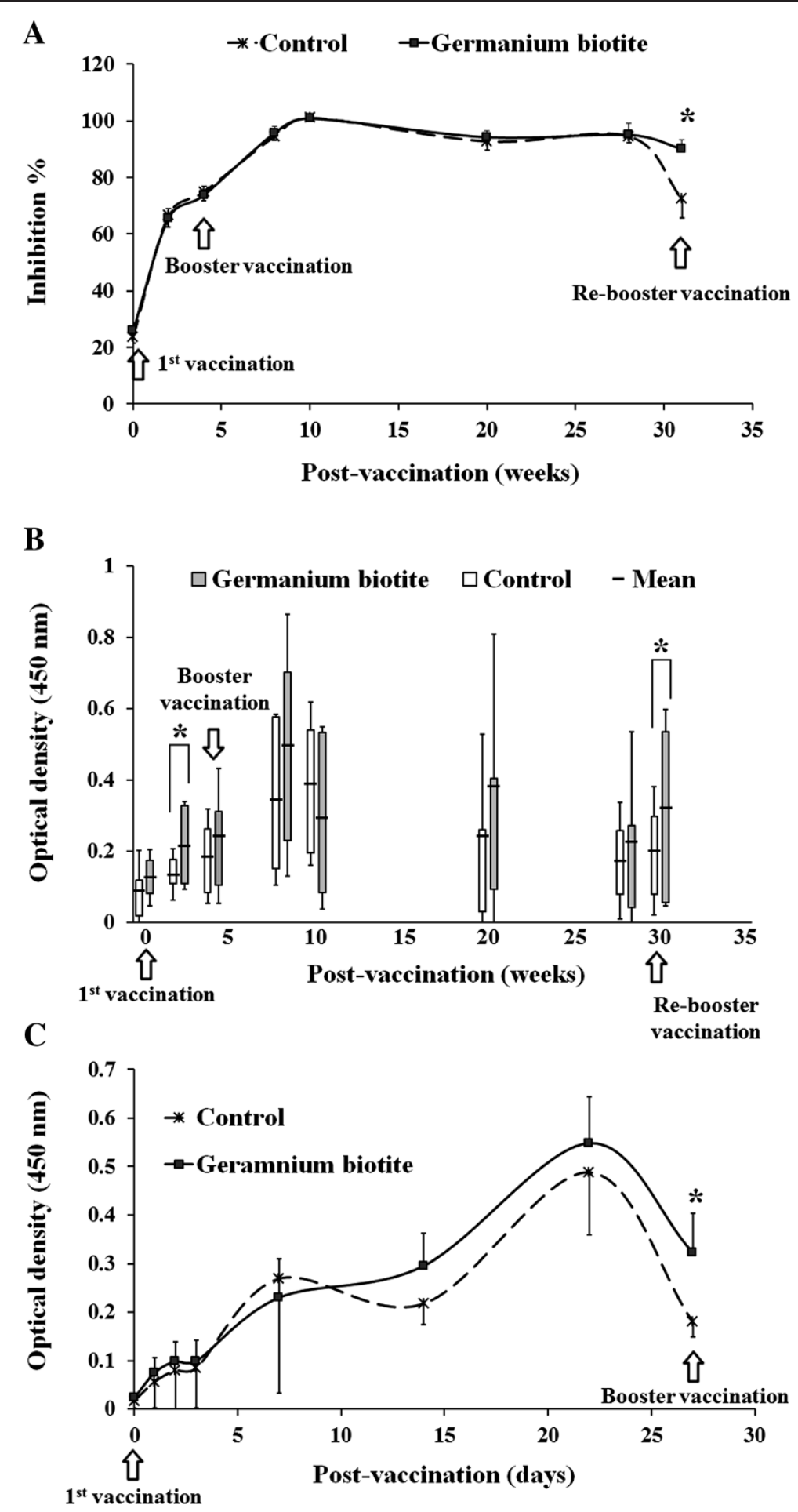

Figure 1 Humoral immune responses after vaccination with Decivac FMD DOE trivalent vaccine in cattle. A. Inhibition percentage (PI) of the antibody against FMDV serotype $\mathrm{O}$ in both the germanium biotite-feeding group and the non-feeding group after vaccination. The high PI value indicates a high level of antibody titer, and the sera that showed more than $50 \%$ of the PI value were considered to be seropositive in the ELISA assays. B. The FMDV serotype O-specific IgA response in the saliva of cattle of the germanium biotite and the control groups after vaccination with Decivac FMD DOE trivalent vaccine. C. Induction of FMDV serotype O-specific lgG in the cattle of the germanium biotite and control groups after vaccination with Decivac FMD DOE trivalent vaccine. 
Table 1 Virus neutralization antibody titers in sera after vaccination

\begin{tabular}{llll}
\hline Group & Samples & 20 weeks & 31 weeks \\
\hline Control & 1 & $<16$ & $<16$ \\
2 & $<16$ & $<16$ \\
3 & $<16$ & $<16$ \\
4 & $<16$ & $<16$ \\
5 & $<16$ & $<16$ \\
6 & $<16$ & $<16$ \\
Germanium biotite & 7 & $<16$ & $<16$ \\
& 1 & $<16$ & 16 \\
& 2 & 16 & $<16$ \\
& 3 & 16 & 32 \\
4 & 16 & 26 \\
& 5 & 16 & $<16$ \\
& 6 & 16 & $<16$ \\
7 & $<16$ & 32 \\
\hline
\end{tabular}

The selected sera were taken from the same cattle in each collection date. All cattle were 6-month-old when the first vaccination was conducted. The sera selected from the germanium biotite group show a higher virus neutralization antibody titer than the control group.

experimental result closely matches with previous studies' findings on the improvement of antibody production by germanium biotite $[18,19]$. Furthermore, germanium biotite feeding showed a high level of IgA in the saliva, which could provide a beneficial effect when it comes to protecting against FMDV infection in cattle [27]. In addition, the IgG response suggested that the point at which to administer the booster injection in the FMD vaccine program for cattle is the same as indicated in previous studies $[6,26]$. However, the increase in antibody duration suggests that the current FMD vaccine program should be reconsidered in animals fed germanium biotite. In this study, sampling of sera after 31 weeks from only first and boosting vaccinated cattle was limited, due to national FMD control policy by which re-booster vaccination carried out every 6 months in cattle. The decline point of PI 6 month after booster vaccination could not be identified. Therefore, the investigation of PI value changes for a longer period should be conducted in the future study to determine the re-booster vaccination timing.

Along with these antibody responses, a VN test was performed using the Andong strain, which is an isolate from the recent outbreak in Korea in 2010. Following the vaccination, 20 weeks was the mid-point of the period showing a high PI value and 31 weeks was the time point that showed a difference in PI values between the two experiment groups. To ensure the reliability of $\mathrm{VN}$ test and reduce the variation, cattle in Farm B were selected and sera of half the number of scale of each group in Farm B were taken from the same cattle in each collection date. As shown in Table 1, all experimental groups showed a low VN titer. These low VN titers might be due to using the heterologous FMDV. The immunological relationship between the $\mathrm{O} 1$ Manisa vaccine and the Andong strains was relatively low or moderate ( $r$-value of approximately 0.3) [28]. A previous study considered an $r$-value in the range of $0.3-1.0$ as indicative of reasonable levels of cross-reactivity [29]. Therefore, the low VN titers observed in this study could be explained by the low level of the $r$-value between the two strains. However, in comparison with the control group, the increase of $\mathrm{VN}$ antibodies in the germanium biotite group suggests that protectivity against FMDV infection might be increased through the supplementation of germanium biotite $[3,6,9]$.

Although the importance of antibodies in protection against FMDV infection is well known [3,6,9], a specific antibody does not guarantee sterile immunity or clinical protection against FMDV infection [9,25]. Moreover, protection against FMDV infection has been observed in the absence of a detectable specific humoral response [30]. Therefore, it is reasonable to consider a possible protective role for innate immune responses in FMD infection and for control of the disease [12,13,26,31]. In this study, high lysozyme activity-indicating macrophage activitywas observed in the germanium biotite group compared to the control group. This result corresponds with the findings of previous studies, which that the macrophage activation effects of silicate $\left(\mathrm{SiO}_{2}\right)$ and aluminosilicate $\left(\mathrm{Al}_{2} \mathrm{SiO}_{5}\right)$, which are major components of germanium

Table 2 Subsets of peripheral blood mononuclear cells after mitogen stimulation

\begin{tabular}{|c|c|c|c|c|c|}
\hline & \multicolumn{2}{|c|}{ Before administration } & \multicolumn{2}{|c|}{23 weeks after administration } & \multirow[t]{2}{*}{ Stimulators } \\
\hline & Control & Germanium biotite & Control & $\overline{\text { Germanium biotite }}$ & \\
\hline$\overline{\text { CD79a }^{+}}$ & $8.40 \%( \pm 0.17)$ & $7.90 \%( \pm 0.14)$ & $6.70 \%( \pm 0.53)$ & $4.70 \%( \pm 1.01)$ & LPS \\
\hline $\mathrm{CD}^{+}$ & $59.50 \%( \pm 0.49)$ & $57.80 \%( \pm 0.53)$ & $93.50 \%( \pm 0.04)$ & $93.10 \%( \pm 0.28)$ & ConA \\
\hline $\mathrm{CD}^{+}$ & $14.10 \%( \pm 0.72)$ & $13.00 \%( \pm 0.22)$ & $15.40 \%( \pm 0.58)$ & $23.80 \%( \pm 0.52)^{* *}$ & ConA \\
\hline $\mathrm{CD}^{+}$ & $12.50 \%( \pm 0.74)$ & $10.40 \%( \pm 1.30)$ & $14.30 \%( \pm 1.40)$ & $10.20 \%( \pm 1.21)$ & ConA \\
\hline $\mathrm{MHC} \mathrm{I}^{+}, \mathrm{II}^{+}$ & $43.00 \%( \pm 0.45)$ & $37.50 \%( \pm 0.12)$ & $8.80 \%( \pm 0.17)$ & $10.20 \%( \pm 0.15)^{* *}$ & ConA \\
\hline
\end{tabular}

The data were expressed as mean \pm standard deviation (SD) $\left({ }^{* *} p<0.01\right.$ compared to the control group at each time point). 


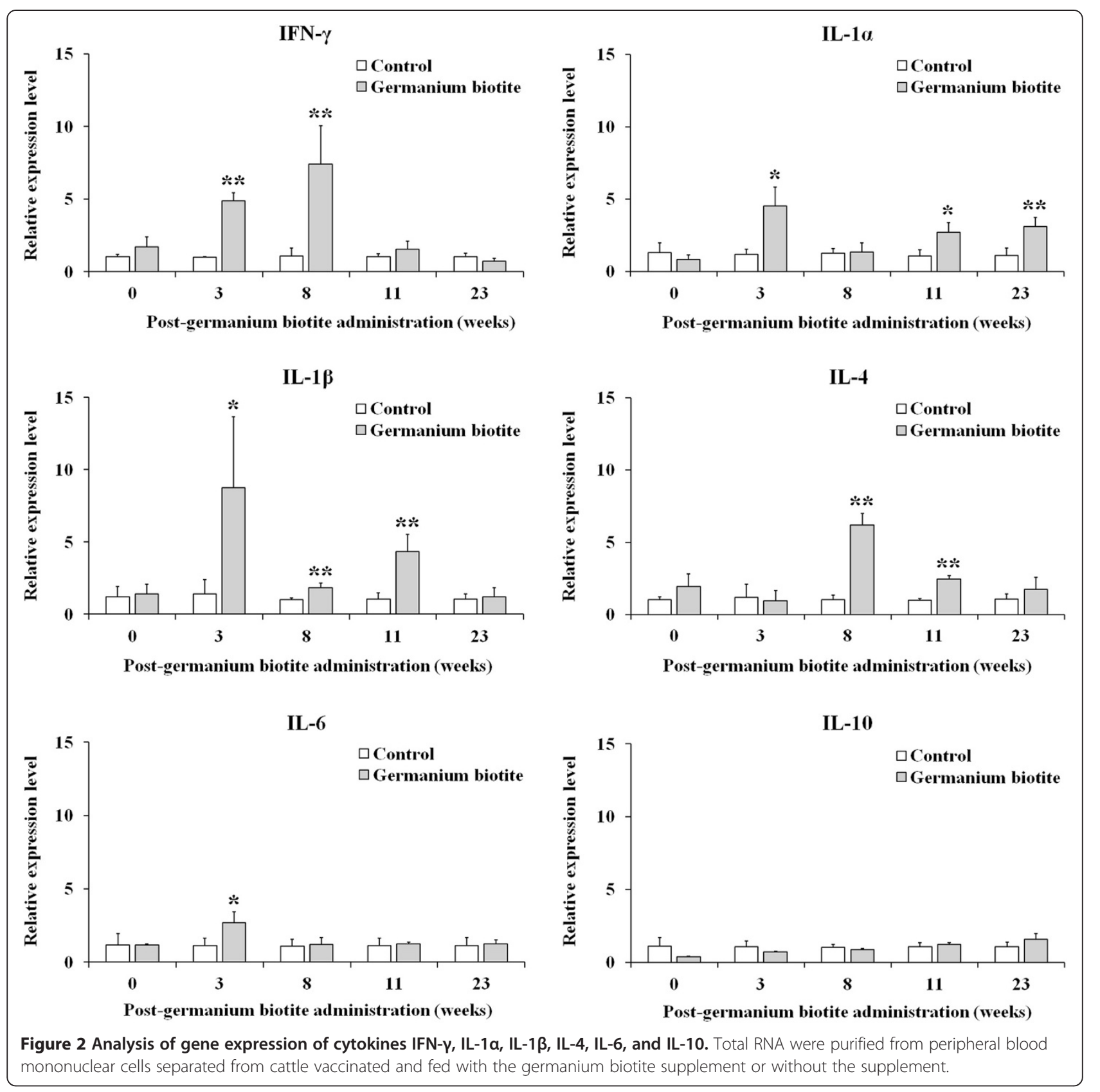

biotite [18,19,21]. Along with macrophage activation, a high proliferative response of $\mathrm{T}$ and $\mathrm{B}$ lymphocytes to the mitogen stimulation was observed in the germanium biotite group compared to the control group, thereby indicating that the marker level of lymphocyte activity in the germanium biotite group was higher than in the control group. Through the analysis of PBMC subset stimulation with ConA and LPS, it can be presumed that the proliferative responses of the $\mathrm{T}$ lymphocytes were predominately associated with $\mathrm{CD}_{4}^{+}$and $\mathrm{MHCI}^{+} \mathrm{II}^{+}$cells, as the $\mathrm{CD}_{4}^{+}$ and $\mathrm{MHCI}^{+} \mathrm{II}^{+}$cells were significantly increased at 23 weeks after stimulation with ConA. The important role of $\mathrm{CD}^{+}{ }^{+} \mathrm{T}$-cells in the induction of the antibody response in ruminants following infection or vaccination with a virus or a viral peptide has been demonstrated [11,12]. Furthermore, MHC II-restricted T-cells (CD4) play an important role in the immune responses to the FMDV antigen and the activation of macrophages [31]. These results show the increase of activation markers for innate immune responses associated with cell-mediated immunity in the germanium biotite group. In addition, previous studies reported that the cross-reactivities against a heterologous virus were mainly induced by $\mathrm{T}$-cell responses to FMDV vaccination [13]. Therefore, it could be postulated 


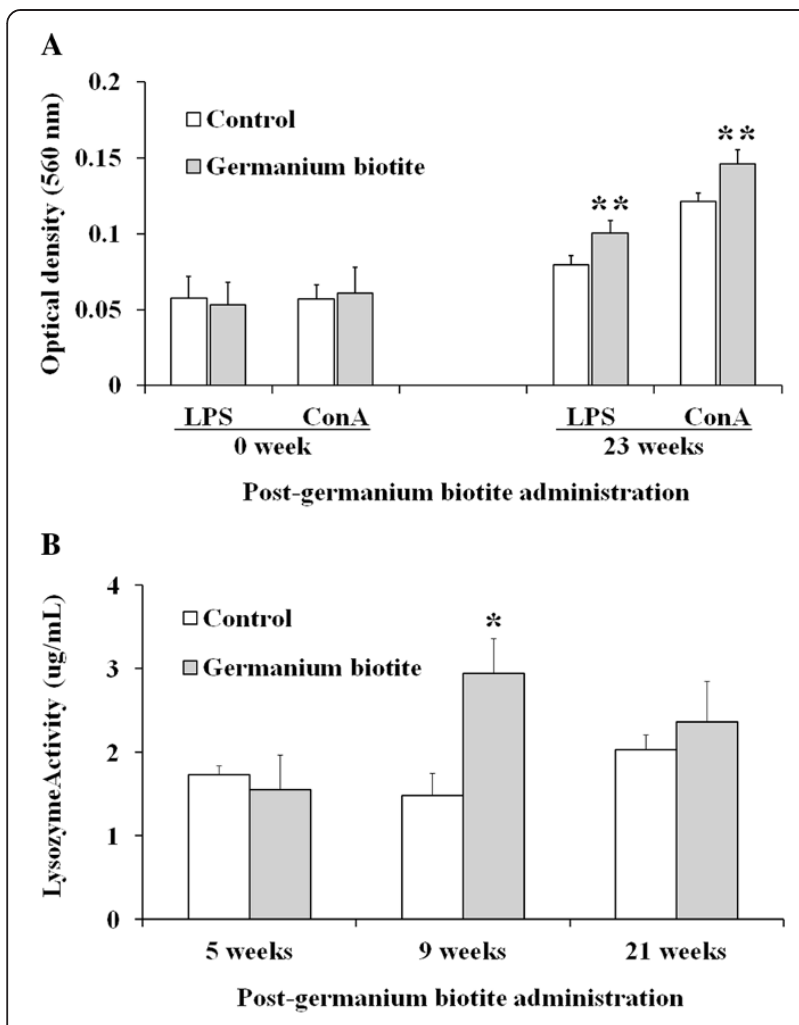

Figure 3 Proliferation of peripheral blood mononuclear cells (PBMCs) and lysozyme activity of sera. A. PBMCs were separated from the vaccinated cattle that were fed with the germanium biotite supplement or without the supplement at 0 and 23 weeks. The proliferation of the PBMCs was estimated after stimulation with LPS or ConA. B. The lysozyme activities of the sera from the vaccinated cattle that were fed with the germanium biotite supplement or without the supplement were measured based on the dissolution ability against Micrococcus lysodeikticus.

that the increase of $\mathrm{VN}$ titer against Andong strain in the germanium biotite group compared to the control might result from enhanced T-cell responses due to germanium biotite feeding.

The increase of the expression of the IFN- $\gamma$, IL- $1 \alpha$, IL$1 \beta$, and IL- 4 genes in the germanium biotite group was in agreement with the results that demonstrated the activation of immune cells associated with cell-mediated immune responses. The increase of the IFN- $\gamma$ expression level and the $\mathrm{CD} 4^{+} \mathrm{T}$-cell subpopulation in our results is closely correlated with a previous report that indicated that $\mathrm{CD} 4^{+} \mathrm{T}$-cells were the major proliferating phenotype and IFN- $\gamma$ producing cells [12]. The induction of IFN- $\gamma$ expression has been observed in antigen-specific T-cell activation [32], MHC II-restricted T-cells (CD4) [5], and activation of macrophages [31]. This immune cell activation induced by IFN- $\gamma$ expression could also be observed in our results through the increases in the $\mathrm{CD}^{+}$and $\mathrm{MHCI}^{+} \mathrm{II}^{+}$cell subpopulations, lysozyme activity, and lymphocyte proliferation level. In addition, the antiviral activity of IFN- $\gamma$ against FMDV contributed to the control of FMDV replication and the spread of the virus within the host via activation of NK cells and macrophages [33]. IL-1 $\alpha$ and IL-1 $\beta$ are mainly produced in activated macrophages and promote Th2 immune cells, which represent the major source of IL-4 [34]. IL-4 also promotes Th2 cell growth and enhances MHC II expression in B-cells [34]. These results suggest that germanium biotite induces Th1 and Th2 responses through the activation of macrophages and CD4+ class II MHCrestricted T-cells $[1,34]$. Considering these results, it can be presumed that supplementation of germanium biotite may activate cell-mediated immunity, thereby enhancing the induction of the immune responses by FMD vaccination and the protectivity against FMDV infection. However, the effects of germanium biotite on T-cell activation through FMDV specific T-cell responses could not be investigated. Therefore, an inquiry on the effects of germanium biotite on immune responses to FMDV vaccination through a comparative study of T-cell responses using FMDV antigen were needed to evaluate the specific effects in a further research.

In addition, a large difference in the appearance of the MHC II subpopulation was observed in the experiment cattle between two time points, before and after the administration of the germanium biotite. Previous studies showed that the MHC II subpopulation in the PBMCs of Holstein at 5-7 and 15-16 months could be estimated at about $32.2 \%$ and $10.7 \%$, respectively [35,36]. Based on these reports, it can be presumed that the significant difference in this study could be caused by the age change in the experiment calves.

\section{Conclusions}

The results of this study suggest an enhancement of the immune responses to FMD vaccination through the supplementation of dietary germanium biotite such as IgG, IgA, and $\mathrm{VN}$ antibodies. It can be presumed that these enhancements are induced by the immunostimulating effects of the germanium biotite, including the activation of macrophages and CD4+ class II MHC-restricted cells, as well as the induction of cytokines, which can activate Th1 and Th2 immune cells. In conclusion, the findings of this study indicate that dietary germanium biotite could be used to improve the efficacy of the FMD vaccine.

\section{Methods}

\section{Experimental animals and vaccination}

This experiment was carried out with 89 cattle from 6-8 months in age (Korean Native Cattle) at three different farms, which were located in different provinces in Korea. Forty-five cattle were raised with feed supplemented with $0.5 \%$ germanium biotite (SoltoB, Seobong 
BioBestech Co, Seoul, Korea; germanium biotite group) from 1 week before vaccination to the end of the experiment, while the rest were raised without supplementation (control group). Control and germanium biotite groups were arranged at each farm (Table 3). All the cattle were intramuscularly vaccinated with $2 \mathrm{ml}$ of Decivac FMD DOE trivalent vaccine (InterVet, Germany). A booster vaccination was given 4 weeks after the first vaccination, according to the national vaccination policy of Korea. Samples of blood, saliva, and feces were collected before vaccination and up to 6 months after the second vaccination. Feces and saliva were collected by swabbing with a cotton swab. Bleeding was carried out from the jugular vein. Sera were collected and stored at $-20^{\circ} \mathrm{C}$ until use after inactivation at $56^{\circ} \mathrm{C}$ for $30 \mathrm{~min}$. The cotton swabs were immersed in $1 \mathrm{ml}$ of PBS for $1 \mathrm{~h}$ at $4^{\circ} \mathrm{C}$, and the supernatants were collected after centrifugation at $1,500 \times \mathrm{g}$ for $15 \mathrm{~min}$ at $4^{\circ} \mathrm{C}$; the supernatants were stored at $-20^{\circ} \mathrm{C}$ and used as samples for the ELISA analysis of FMDVspecific IgA.

The germanium biotite consisted of silicon dioxide (61.90\%), aluminum dioxide (23.19\%), iron oxide (3.97\%), sodium oxide $(3.36 \%)$, calcium oxide $(<2 \%)$, magnesium oxide $(<2 \%)$, titanium oxide $(<2 \%)$, and $36 \mathrm{ppm}$ germanium. All procedures in the animal experiment in this study were approved by the Institution Animal Care and Use Committee (IACUC) of Seoul National University (SNU-111210-1).

\section{Measurement of antibody against FMDV}

The antibody titers against FMDV were measured using the PrioCHECK FMDV type O ELISA kit (Prionics, Switzerland) following the manufacturer's protocols. The serotype $\mathrm{O}$ antibody titer using this ELISA kit is the criterion for assessing seropositivity in the national policy of Korea. The antibody titer against FMDV was indicated by the PI value. The high PI value presents a high level of antibody titer, and the sera that showed more than $50 \%$ of the PI value were considered to be seropositive in the ELISA assays. FMDV-specific IgG in the serum were measured using the PrioCHECK FMDV type O ELISA kit (Prionics) with 1:40 diluted serum and horseradish peroxidase (HRP)-conjugated sheep antibovine IgG (Bethyl, USA). In addition, FMDV-specific IgA in the saliva and feces was measured using the PrioCHECK FMDV type O ELISA kit (Prionics) with $1 \mathrm{x}$ samples extracted by PBS from cotton swabs and HRPconjugated rabbit anti-bovine IgA (Bethyl). All procedures for detecting FMDV-specific IgG and IgA followed the manufacturer's protocols with a 1:3,000 dilution of each immunoglobulin-specific conjugate.

\section{Analysis of $\mathrm{VN}$ antibody titer}

The VN titer was measured in eight randomly selected sera from the germanium biotite and seven sera from the control groups of Farm B at 20 and 31 weeks after vaccination (Tables 1 and 3). Fifty microliters of twofold serially diluted bovine sera were reacted with $50 \mu \mathrm{l}$ of the FMDV Andong strain $\left(100 \mathrm{TCID}_{50}\right)$, a serotype O FMDV Korean isolate from the 2010 outbreak, at $37^{\circ} \mathrm{C}$ for $1 \mathrm{~h}$. After incubation, $50 \mu \mathrm{l}$ of complete Dulbecco's Modified Eagle Medium (Gibco, USA) with $5 \%$ fetal bovine serum containing $1 \times 10^{4}$ LFBK cells $/ \mathrm{ml}$ was added to the reaction mixture. After incubation of the reaction mixtures at $37^{\circ} \mathrm{C}$ for $48 \mathrm{~h}$, the $\mathrm{VN}$ titer was determined as the final dilution of serum showing no cytopathic effect.

\section{Analysis of PBMC subsets}

Among the 89 cattle, analysis of the peripheral lymphocyte profiles was conducted with ten Korean native cattle (IACUC permission no. SNU-111210-1). Five of them were fed with a commercial feed supplemented with $0.5 \%$ germanium biotite (germanium biotite group), while the rest were fed without the supplement (control group). PBMCs were cultured from the heparinized whole blood of the experiment cattle using the Histopaque ${ }^{\circ}-1077$ Hybri-Max ${ }^{\text {mi }}$ (Sigma, USA) system, as described by the manufacturer. The PMBCs were then stimulated with DPBS (Gibco), ConA (5 g/ml, Sigma) for CD3, CD4, CD8, and MHC I and II and LPS (5 g/ml, Sigma) for CD79a staining for $96 \mathrm{~h}$

Table 3 Experiment design for grouping, sampling, and analysis

\begin{tabular}{lllll}
\hline Farms & \begin{tabular}{l} 
Number of cattle \\
\cline { 2 - 3 } Control
\end{tabular} & Germanium biotite & Analysis of \\
\hline A & 26 & 22 & Serum, saliva, feces & Inhibition percentage, ELISA for antibody levels (IgG and IgA), Iysozyme activity \\
B & 13 & 18 & Serum, saliva, feces & $\begin{array}{l}\text { Inhibition percentage, ELISA for antibody levels (IgG and IgA), lysozyme activity, } \\
\text { virus neutralization test }\end{array}$ \\
C & 5 & 5 & Serum, saliva, feces, PBMC & $\begin{array}{l}\text { Inhibition percentage, ELISA for antibody levels (IgG and IgA), lysozyme activity, } \\
\text { PBMC (phenotype, cytokines, proliferation) }\end{array}$
\end{tabular}

Among 89 cattle at $6-8$ months of age, 45 cattle were raised with feeds supplemented with $0.5 \%$ germanium biotite from 1 week before vaccination to the end of experiment (germanium biotite group) and the rest of them were raised without supplementation (control group). 
Table 4 Antibodies used to detect the cell surface markers

\begin{tabular}{lll}
\hline Target & Antibody & Company \\
\hline CD79a & PE-conjugated mouse anti-bovine CD79a & Abcam, UK \\
CD3 & FITC-conjugated hamster anti-bovine CD3 & Abcam \\
CD4 & PE-conjugated mouse anti-bovine CD4 & US Biological, USA \\
CD8 & PE-conjugated mouse anti-bovine CD8 & LifeSpan BioSciences, USA \\
MHC I & FITC-conjugated mouse anti-bovine MHC I & Novus Biological, USA \\
MHC II & Mouse anti-bovine MHC II & Abcam \\
FC receptor blocking & Polyclonal mouse lgG fraction & Abcam \\
& Monoclonal Armenian hamster lgG fraction & Abcam \\
\hline
\end{tabular}

${ }^{a}$ Mouse anti-bovine MHC II antibody was conjugated with PE using an EasyLink B-Phycoerythrin conjugation kit (Abcam).

to characterize the phenotype of PBMC responding to stimulation with mitogens. The procedures for cell fixation and antibody reaction to detect the cell markers were performed as previously reported [37] using the DPBS with $0.2 \%$ Tween $^{\oplus} 20$ (Sigma) as the permeabilisation solution. To prevent a non-specific reaction of the Fc receptor with the detection antibodies, the fixed cells were reacted with normal Ig from the antibody-producing host for $10 \mathrm{~min}$ and reacted with fluorescein isothiocyanate (FITC) or phycoerythrin (PE)-conjugated cell surface marker detection antibodies. After the reaction, the number of cells reacting with the FITC or PE conjugate was analyzed using Flow cytometric analysis (BD, USA). The monoclonal antibodies used for the staining of the cells surface markers are listed in Table 4.

\section{Analysis of cytokine gene expression in PBMC}

Total RNAs were extracted from PBMCs with the RNeasy mini kit (Qiagen, USA), and cDNAs were synthesized with the reverse transcription kit (Qiagen). The relative expression levels of IFN- $\gamma$, IL- $1 \alpha$, IL- $1 \beta$, IL-4, IL- 6 , and IL-10 were quantitated using the Rotor-Gene SYBR Green PCR kit (Qiagen) and Rotor-Gene Q (Qiagen), as previously reported [38]. The expression levels were normalized by $\beta$ actin expression using the $2^{-\Delta \Delta C}$ method [39].

\section{Analysis of PBMC proliferation}

A lymphocyte proliferation assay was conducted with the PBMCs collected from the experiment cattle. Ninety microliters of PBMCs $\left(5 \times 10^{6}\right.$ cells $\left./ \mathrm{ml}\right)$ in a 96 -well plate were incubated with ConA $(5 \mu \mathrm{g} / \mathrm{ml})$ and LPS $(5 \mu \mathrm{g} / \mathrm{ml})$ at $37^{\circ} \mathrm{C}$ for $96 \mathrm{~h}$ in a $5 \% \mathrm{CO}_{2}$ atmosphere. Absorbance at $560 \mathrm{~nm}$ was measured $2 \mathrm{~h}$ after the addition of $10 \mu \mathrm{l}$ of $10 \times$ Prestoblue (Invitrogen, USA). Proliferation of the PBMCs was compared based on this absorbance.

\section{Analysis of lysozyme activities}

Lysozyme activities in the serum were measured based on the dissolution ability against Micrococcus lysodeikticus
(Sigma) [40]. The activity was calculated based on the standard curve generated from the serially diluted lysozyme solution.

\section{Statistical analysis}

The data were expressed as mean \pm standard deviation (SD). A student's $t$-test and repeated measures of ANOVA were performed for statistical analysis of the data. All statistical analyses of data were carried out using SPSS version 19.0 software (SPSS, USA). A probability value of $p<0.05$ was considered significant.

\section{Abbreviations}

ConA: Concanavalin A; FITC: Fluorescein isothiocyanate; FMD: Foot-andmouth disease; FMDV: Foot-and-mouth disease virus; HRP: Horseradish peroxidase; IACUC: Institution Animal Care and Use Committee; LPS: Lipopolysaccharide; MHC: Major histocompatibility complex; PBMC: Peripheral blood mononuclear cell; PE: Phycoerythrin; PI: Inhibition percentage; SAT: South African territories; Th1: T helper type 1; Th2: T helper type 2; VN: Virus neutralization.

\section{Competing interests}

Effects of the germanium biotite in FMD vaccination are applying to patent in Korea.

\section{Authors' contributions}

MJ was involved in sampling, sample processing, data analysis and drafting of manuscript. MKS was involved in sampling and participated in the studies of ELISA for antibodies. SBC and SWS carried out sampling and participated in study of cytokine. AY carried out sampling. WJL and HTP participated in the analysis of PBMC and in study of cytokines analysis. JHP and BK carried out virus neutralization test with a Korea isolate of FMDV. YKJ carried out development of germanium biotite. HSY was involved in experimental design and supervision of the experiments and manuscript revision. All authors read and approved the final manuscript.

\section{Acknowledgements}

This study was supported by Ministry of Agriculture, Food and Rural Affairs (IPET 111104-1), BK21 for Veterinary Science and Research Institute for Veterinary Science, Seoul National University, Korea.

\section{Author details}

'Department of Infectious Diseases, College of Veterinary Medicine, Seoul National University, Seoul 151-742, South Korea. ${ }^{2}$ Foot and Mouth Disease Division, Animal and Plant Quarantine Agency, Anyang, Gyeonggido 430-757, South Korea. ${ }^{3}$ Seobong BioBestech Co., Ltd, Hyechon Bldg. \#401, 831 Yeoksam-dong, Kangnam-gu, Seoul 135-792, South Korea. ${ }^{4}$ Institute of Green Bio Science and Technology, Seoul National University, Pyeongchang 232-916, South Korea. 
Received: 16 April 2014 Accepted: 4 August 2014

Published online: 12 August 2014

\section{References}

1. Sobrino F, Sáiz M, Jiménez-Clavero MA, Núñez Jl, Rosas MF, Baranowski E, Ley V: Foot-and-mouth disease virus: a long known virus, but a current threat. Vet Res 2001, 32:1-30.

2. OIE: OIE Terrestrial Manual 2012. Paris: OIE; 2011.

3. Cox SJ, Barnett PV: Experimental evaluation of foot-and-mouth disease vaccines for emergency use in ruminants and pigs: a review. Vet Res 2009, 40:13-42.

4. Nagendrakumar SB, Srinivasan VA, Madhanmohan M, Yuvaraj S, Parida S, D Nardo A, Horsington J, Paton DJ: Evaluation of cross-protection between $\mathrm{O}_{1}$ Manisa and $\mathrm{O}_{1}$ Campos in cattle vaccinated with foot-and-mouth disease virus vaccine incorporating different payloads of inactivated $\mathrm{O}_{1}$ Manisa antigen. Vaccine 2011, 29:1906-1912.

5. Parida S: Vaccination against foot-and-mouth disease virus: strategies and effectiveness. Expert Rev Vaccines 2009, 8:347-365.

6. Park JH: Requirements for improved vaccines against foot-and-mouth disease epidemics. Clin Exp Vaccine 2013, 2:8-18.

7. Elnekave E, Li Y, Zamir L, Even-Tov B, Hamblin P, Gelman B, Hammond J, Klement $E$ : The field effectiveness of routine and emergency vaccination with an inactivated vaccine against foot and mouth disease. Vaccine 2013, 31:879-885.

8. Höhlich BJ, Wiesmüller KH, Haas B, Gerner W, Correa R, Hehnen HR, Schlapp $T$, Pfaff E, Saalmüller A: Induction of an antigen-specific immune response and partial protection of cattle against challenge infection with footand-mouth disease virus (FMDV) after lipopeptide vaccination with FMDV-specific B-cell epitopes. J Gen Virol 2003, 84:3315-3324.

9. McCullough KC, Bruckner L, Schaffner R, Fraefel W, Müller HK, Kihm U: Relationship between the anti-FMD virus antibody reaction as measured by different assays, and protection in vivo against challenge infection. Vet Microbiol 1992, 30:99-112

10. Li R, Sakwiwatkul K, Yutao L, Hu S: Enhancement of the immune responses to vaccination against foot-and-mouth disease in mice by oral administration of an extract made from Rhizoma Atractylodis Macrocephalae (RAM). Vaccine 2009, 27:2094-2098.

11. Gerner W, Carr BV, Wiesmüller KH, Pfaff E, Saalmüller A, Charleston B: Identification of a novel foot-and-mouth disease virus specific T-cel epitope with immunodominant characteristics in cattle with MHC serotype A31. Vet Res 2007, 38:565-572.

12. Oh Y, Fleming L, Statham B, Hamblin P, Barnett P, Paton DJ, Park JH, Joo YS, Parida S: Interferon- $\gamma$ induced by in vitro re-stimulation of CD4+ T-cells correlates with in vivo FMD vaccine induced protection of cattle against disease and persistent infection. PLoS One 2012, 7:e44365.

13. Doel T: Natural and vaccine induced immunity to FMD. Curr Top Microbiol Immunol 2005, 288:103-131.

14. Oh Y, Reid S, Cox S, Statham R, Mahapatra M, Anderson J, Barnett P, Charleston B, Paton D, Parida S: Interferon- $\gamma$ production in vitro from whole blood of foot-and-mouth disease virus (FMDV) vaccinated and infected cattle after incubation with inactivated FMDV. Vaccine 2006, 24:964-969.

15. Guzman E, Taylor G, Charleston B, Skinner MA, Ellis SA: An MHC-restricted CD8+ T-cell response is induced in cattle by foot-and-mouth disease virus (FMDV) infection and also following vaccination with inactivated FMDV. J Gen Virol 2008, 89:667-675.

16. Grubman MJ, Moraes MP, Segundo DS, Pena L, De Los Santos T: Evading the host immune response: how foot-and-mouth disease virus has become an effective pathogen. FEMS Immunol Med Microbiol 2008, 53:8-17.

17. Summerfield A, Guzylack-Piriou L, Harwood L, McCullough KC: Innate immune responses against foot-and-mouth disease virus: current understanding and future directions. Vet Immunol Immunopatho/ 2009, 128:205-210.

18. Jung BG, Toan NT, Cho SJ, Ko J, Jung YK, Lee BJ: Dietary aluminosilicate supplement enhances immune activity in mice and reinforces clearance of porcine circovirus type 2 in experimentally infected pigs. Vet Microbiol 2010, 143:117-125.

19. Jung $B G$, Lee $J A$, Lee $B$ J: Antiviral effect of dietary germanium biotite supplementation in pigs experimentally infected with porcine reproductive and respiratory syndrome virus. J Vet Sci 2013, 14:135-141.
20. Jung M, Jung BG, Cha SB, Shin MK, Lee WJ, Shin SW, Lee JA, Jung YK, Lee BJ, Yoo HS: The effects of germanium biotite supplement as a prophylactic agent against respiratory infection in calves. Pak Vet J 2012, 32:319-324.

21. Holian A, Uthman MO, Goltsova T, Brown SD, Hamilton RF Jr: Asbestos and silica-induced changes in human alveolar macrophage phenotype. Environ Health Perspect 1997, 105:1139-1142.

22. Aikoh T, Tomokuni A, Matsukii T, Hyodoh F, Ueki H, Otsuki T, Ueki A: Activation-induced cell death in human peripheral blood lymphocytes after stimulation with silicate in vitro. Int J Oncol 1998, 12:1355-1359.

23. Mollick JA, Cook RG, Rich RR: Class II MHC molecules are specific receptors for staphylococcus enterotoxin A. Science 1989, 244:817-820

24. Park JH, Lee KN, Ko YJ, Kim SM, Lee HS, Shin YK, Sohn HJ, Park JY, Yeh JY, Lee YH: Control of foot-and-mouth disease during 2010-2011 epidemic, South Korea. Emerg Infect Dis 2013, 19:655-659.

25. Zheng H, Guo J, Jin Y, Yang F, He J, Lv L, Zhang K, Wu Q, Liu X, Cai X: Engineering foot-and-mouth disease viruses with improved growth properties for vaccine development. PLoS One 2013, 8:e55228.

26. Cox SJ, Aggarwal N, Statham RJ, Barnett PV: Longevity of antibody and cytokine responses following vaccination with high potency emergency FMD vaccines. Vaccine 2003, 21:1336-1347.

27. Doel T: Optimisation of the immune response to foot-and-mouth disease vaccines. Vaccine 1999, 17:1767-1771.

28. Park JN, Lee SY, Chu JQ, Lee YJ, Kim RH, Lee KN, Kim SM, Tark DS, Kim B, Park JH: Protection to homologous and heterologous challenge in pigs immunized with vaccine against foot-and-mouth disease type $O$ caused an epidemic in East Asia during 2010/2011. Vaccine 2014, 32:1882-1889.

29. Barnett P, Samuel A, Statham R: The suitability of the 'emergency' footand-mouth disease antigens held by the International Vaccine Bank within a global context. Vaccine 2001, 19:2107-2117.

30. Sanz-Parra A, Jimenez-Clavero MA, Garcla-Briones MM, Blanco E, Sobrino F, Ley $\mathrm{V}$ : Recombinant viruses expressing the foot-and-mouth disease virus capsid precursor polypeptide (P1) induce cellular but not humoral antiviral immunity and partial protection in pigs. Virology 1999, 259:129-134.

31. Boehm U, Klamp T, Groot M, Howard J: Cellular responses to interferon- $\gamma$. Annu Rev Immunol 1997, 15:749-795.

32. Carr BV, Lefevre EA, Windsor MA, Inghese $C$, Gubbins $S$, Prentice $H$, Juleff $\mathrm{ND}$, Charleston B: CD4+ T-cell responses to foot-and-mouth disease virus in vaccinated cattle. J Gen Virol 2013, 94:97-107.

33. Zhang Z, Hutching G, Kitching P, Alexandersen S: The effects of gamma interferon on replication of foot-and-mouth disease virus in persistently infected bovine cells. Arch Virol 2002, 147:2157-2167.

34. Elgert KD: Immunolgy. 2nd edition. Hoboken: John Wiley \& Sons Inc:; 2009.

35. Griebel PJ, Qualtiere L, Davis WC, Lawman MJP, Babiuk LA: Bovine peripheral blood leukocyte subpopulation dynamics following a primary bovine herpesvirus-1 infection. Viral Immunol 1987, 1:267-286.

36. Burton JL, Kehrli ME: Effects of dexamethasone on bovine circulating $T$ lymphocyte populations. J Leukoc Biol 1996, 59:90-99.

37. Gerner W, Käser T, Pintarič M, Groiß S, Saalmüller A: Detection of intracellular antigens in porcine PBMC by flow cytometry: A comparison of fixation and permeabilisation reagents. Vet Immunol Immunopathol 2008, 121:251-259

38. Konnai S, Usui T, Ohashi K, Onuma M: The rapid quantitative analysis of bovine cytokine genes by real-time RT-PCR. Vet Microbiol 2003, 94:283-294.

39. Livak KJ, Schmittgen TD: Analysis of relative gene expression data using real-time quantitative PCR and the $2^{-\Delta \Delta C T}$ method. Methods 2001, 25:402-408

40. Kreukniet MB, Nieuwland MGB, Van der Zijpp AJ: Phagocytic activity of two lines of chickens divergently selected for antibody production. Vet Immunol Immunopathol 1994, 44:377-387.

\section{doi:10.1186/s12917-014-0179-6}

Cite this article as: Jung et al.: Supplementation of dietary germanium biotite enhances induction of the immune responses by foot-andmouth disease vaccine in cattle. BMC Veterinary Research 2014 10:179. 